\title{
EXCITATION-ENHANCED DISLOCATION MOBILITY IN SEMICONDUCTORS
}

\author{
G. VANDERSCHAEVE, C. LEVADE, A. FARESS, J.J. COUDERC and D. CAILLARD* \\ Laboratoire de Physique des Solides, INSA Complexe Scientifique de Rangueil, F-31077 \\ Toulouse Cedex, France \\ ${ }^{*}$ CEMES/LOE/CNRS, BP. 4347, F-31055 Toulouse Cedex, France
}

\begin{abstract}
Quantitative features of the enhancement effect by either laser light illumination or electron irradiation on the dislocation motion in semiconductors are surveyed. The experiments show that moving dislocations experience a lattice friction which is lowered under excitation. Theoretical analysis based on the kink diffusion model for dislocation motion indicates that the observed reduction in activation energy corresponds to the electronic energy level in the band gap associated with the straight dislocation sites.
\end{abstract}

\section{INTRODUCTION}

It was demonstrated in recent years that plastic properties and related dislocation behaviour in semiconductors may be very sensitive to light illumination (photoplastic effect) or electron beam irradiation (cathodoplastic effect). For example, the flow stress of II-VI compounds, both with the sphalerite and wurtzite structures, is drastically increased with illumination of light near the absorption edge $[1,2]$. In contrast to this hardening effect, a marked enhancement of the dislocation velocity (equivalent to a softening) is observed in large band gap semiconductors (both elemental and compound) as the result of either laser light illumination or electron beam irradiation. This particular behaviour, so-called radiation-enhanced dislocation motion, reflects the special properties of dislocations in semiconductors, as compared with those in metals : they provide electronic energy levels in the band gap and act as active centers for electronic processes in the crystals. Consequently, the dislocation velocity in semiconductors is known to be affected by doping of electrically active impurities [3]. In the same way, excitation effects on the dislocation mobility are considered to be associated with electronic states of dislocations. These effects have been interpreted in terms of enhanced defect motion, due to the recombination of injected carriers at the dislocation sites $[4,5]$. It is therefore expected that the study of the dislocation behaviour under either electronic or photonic excitations can give some information on the electronic states associated with dislocations in these materials.

On the other hand the growing interest for such investigations is caused by the fact that these effects underlie the degradation of some optoelectronic devices. Indeed it has been reported that the cause of their rapid degradation is the multiplication of dislocations during the operation of the device [6]. Therefore a detailed study of the excitation-enhanced dislocation motion under well defined conditions is expected to give a deeper insight into the nature of ageing phenomena in semiconductor devices.

This article will focus on the influence of the irradiation by electron beam or laser light on the dislocation glide under applied stress in semiconductors. A review of experimental features will be 
given, with special emphasis on new results obtained at a microscopic scale by the present authors using in situ deformation experiments in a transmission electron microscope. These experiments show that the dislocation mobility is enhanced as a result of the reduction in the activation energy for dislocation motion. Then, a microscopic mechanism, as recently proposed by Maeda and Takeuchi [7] will be presented. The origin of this energy reduction is discussed on the basis of the kink diffusion mechanism for dislocation glide in semiconductors (Peierls regime) [8] : the dislocation motion is assisted by the energy released upon non radiative recombination of electron-hole pairs at the defect. For a better understanding of the paper, a brief summary of the properties of dislocations in semiconductors will be first given.

\section{PROPERTIES OF DISLOCATIONS IN SEMICONDUCTORS. A BRIEF REVIEW}

At low temperature, dislocations with Burgers vector $1 / 2<110\rangle$ lie along $\langle 110\rangle$ rows of the $\{111\}$ glide planes, due to covalent bonding inducing a high Peierls friction. Therefore a glide loop possesses two types of segments, screw and $60^{\circ}$ ones. In compound semiconductors, a complication arises in the description of dislocations, from the existence of two types of atoms. Depending on the nature of atoms ending the extra half plane we have to distinguish two types of $60^{\circ}$ dislocations in compound semiconductors, namely $\alpha$ and $\beta$ ones [9]. Dislocations are generally dissociated into $1 / 6<112>$ partials bounding a stacking fault : as a result, partials are either $30^{\circ}$ or $90^{\circ}$ in character.

In elemental semiconductors, a slight but significant difference in the mobility of $60^{\circ}$ dislocations has been observed, depending on the character $\left(30^{\circ}\right.$ or $\left.90^{\circ}\right)$ of the leading partial $[10,11]$. In undoped III-V compounds, $\alpha$ dislocations are much more rapid than $\beta$ and screw dislocations, that have similar mobilities [12-15]; this has been attributed to the lowest mobility of the $30^{\circ} \beta$ partial $[13,16]$. The degree of asymmetry in dislocation velocity appears to be lower in II-VI compounds [1, 17].

In materials experiencing a high lattice friction such as covalent and ionocovalent materials, dislocation glide consists of two elementary steps [8] : the initial kink pair formation and the subsequent kink migration along the dislocation line. Depending on the mobility of kinks, two limiting cases are considered :

(i) For slow kink motion and/or long moving rectilinear dislocation segment, the kink collides and annihilates with another kink of opposite sign generated on the same segment. The dislocation velocity $\mathrm{v}$ is proportional to the mean free path of kinks, $\mathrm{X}$ :

$$
v=h X J=2 h \sqrt{J v_{k}}
$$

where $J$ is the rate of nucleation of kink pairs per unit length of dislocation, $h$ the distance between adjacent Peierls valleys and $v_{k}$ the kink velocity. We have [8] :

$$
\begin{gathered}
v_{k}=\nu_{k} a^{2} \frac{\tau b h}{k T} \exp -\frac{W_{m}}{k T} \\
J=\nu_{d} \frac{\tau b h}{k T} \exp -\frac{F_{k p}+W_{m}}{k T}
\end{gathered}
$$

with $\nu_{\mathrm{k}}\left(\nu_{\mathrm{d}}\right)$ being the trial frequency of kink migration (kink formation), a the periodicity along the dislocation line (here equal to $b$ the Burgers vector), $\tau$ the applied stress, $W_{m}$ the activation energy for kink migration and $F_{k p}(\tau)$ the activation energy for nucleation of a kink pair. Assuming $\nu_{\mathrm{k}}=\nu_{\mathrm{d}}=\nu_{\mathrm{o}}$ (Debye frequency), we have :

$$
\mathrm{v}=2 \nu_{\mathrm{o}} \frac{\tau \mathrm{b}^{2} \mathrm{~h}^{2}}{\mathrm{kT}} \exp -\frac{\mathrm{F}_{\mathrm{kp}} / 2+\mathrm{W}_{\mathrm{m}}}{\mathrm{kT}}
$$


(ii) For fast kink motion and/or short moving rectilinear segments of length $L$, no zautal amnihilation of kinks takes place and the dislocation velocity is :

$$
\begin{gathered}
\mathrm{v}=\mathrm{h} \mathrm{L} J \\
\mathrm{v}=\nu_{\mathrm{o}} \frac{\tau \mathrm{bh}^{2}}{\mathrm{kT}} \mathrm{L} \exp -\frac{\mathrm{F}_{\mathrm{kp}}+\mathrm{W}_{\mathrm{m}}}{\mathrm{kT}}
\end{gathered}
$$

Therefore two regimes of dislocation mobilities have to be distinguished depending on whether the length of the moving dislocation segment $(\mathrm{L})$ is much larger, or much smaller than the mean free path of kinks (X). In the latter case, $v$ is proportional to $\mathrm{L}$ ("length-effect" regime) whereas in the former one, it is length-independent. Which condition is actually realized under most experimental conditions is often speculative.

Under excitation, elementary processes of dislocation motion are considered to be basically the same as those in darkness (see paragraph 4). Indeed, experimental features are consistent with a dislocation glide controlled by the Peierls mechanism, even under excitation.

\section{EXPERIMENTAL FEATURES OF EXCITATION-ENHANCED DISLOCATION MOBILITIES IN SEMICONDUCTORS}

The chapter is devoted to a review of the main results obtained using different experimental techniques on the mobilities of dislocations under excitation in a variety of semiconductors. We will describe in some details experiments showing that in most compounds, photonic or electronic excitations led to an enhancement of dislocation velocity. The case of II-VI compounds is more complicated. Although these compounds present a positive photoplastic effect (i.e. an hardening under light illumination), the dislocation velocity is strongly enhanced under electron irradiation (see paragraph 3.2.). The behaviour of dislocations under photonic excitation in II-VI compounds appears to be very different from those described here and will not be discussed further.

Commonly used techniques for studying the velocities of dislocations in semiconductors are double-etching technique, scanning electron microscopy in the cathodoluminescence mode and in situ straining experiments in a transmission electron microscope. All these techniques are suitable for quantitative measurements but the poor spatial resolution of the two former ones is not sufficient to characterize unambiguously the microscopic processes affected by excitations. In this respect, transmission electron microscopy has some advantages :

(i) In situ straining experiments in a transmission electron microscope makes it possible to study the dislocation motion in the first stages of source working, i.e. for short mobile segments. This point is very important since as will be shown in paragraph 4, it makes it possible to discriminate between the elementary processes responsible for the excitation-enhanced dislocation glide.

(ii) The local shear stress acting on the moving dislocations can be evaluated by measuring their radii of curvature.

Nevertheless, as most experimental results have been obtained from semi-microscopical observations, we shall start in describing the corresponding experiments.

\subsection{Semi-microscopical observations of excitation-enhanced dislocation glide}

Illumination enhancement of dislocation mobilities has been studied using the double-etching technique in Si samples strained in compression [18] and in GaAs samples subjected to a bending stress [19]. On the other hand considerable work has been devoted to the electron-irradiation enhanced dislocation motion by Maeda, Takeuchi and coworkers [4, 5, 20-21], using in situ cathodoluminescence 
scanning electron microscopy. In this technique, dislocations appearing at the specimen surface are observed as dark spots. Samples are subjected to a bending stress and the induced dislocation movement is seen as a displacement of black dots. This technique has been applied to III-V compounds GaAs $[4,5]$ and GaP [21]. Cathodoplastic effects in Si [22] and InP [5] have been studied using scanning electron microscopy by the same group using a somewhat different technique : dislocation mobilities have been measured by the double-etching technique.

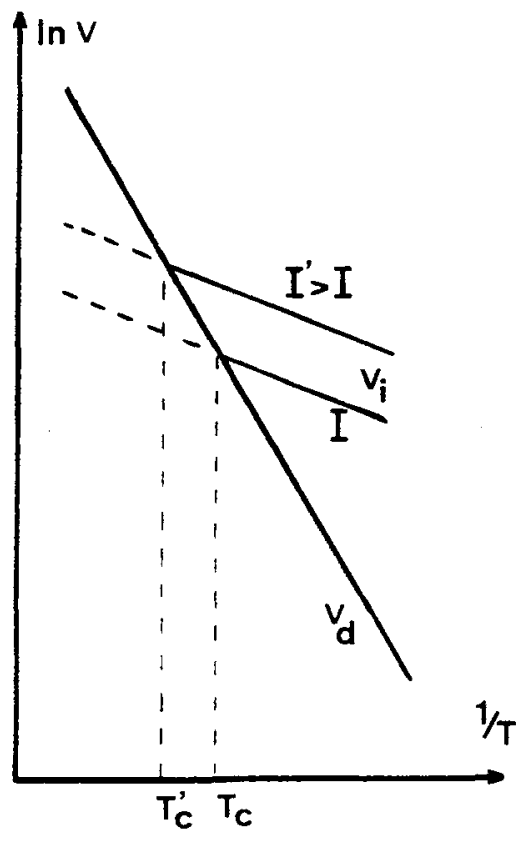

Fig. 1 : Experimental features of excitation effects on dislocation velocity (schematic)

All these measurements have shown experimental features in common though no effect was observed in $\mathrm{Ge}$ [22]. The dislocation velocities under $\left(v_{\mathbf{i}}\right)$ and without $\left(v_{d}\right)$ excitation versus the reciprocal temperature are schematically depicted in fig. 1. A summary of experimental features is given below :

1) Enhancement is observed below a critical temperature $T_{c}$.

2) The influence of excitation is reversible.

3) Either under excitation or without excitation, the dislocation velocity obeys a general Arrhenius law; both the preexponential factor and the activation energy are reduced under excitation (Fig. 1).

4) The increase of dislocation velocity varies linearly with the intensity of the excitation but as will be shown in next section a saturation of the effect is observed for high electron beam intensities.

5) The activation energy under excitation does not depend on the intensity I (Fig. 1).

Thus the dislocation velocity under excitation may be expressed as :

$\mathrm{v}=\mathrm{v}_{\mathrm{od}} \exp -\frac{\mathrm{E}_{\mathrm{d}}}{\mathrm{k} T}+\mathrm{v}_{\mathbf{o i}}^{\prime} \mathrm{I} \exp -\frac{\mathrm{E}_{\mathrm{i}}}{\mathrm{kT}}$

where $E_{d}$ is the activation energy in the dark (without excitation, i.e. $I=0$ ) and $E_{j}=E_{d}-\Delta E$ the apparent activation energy under excitation below $T_{c}$. At high temperature the first term dominates so that no effect of excitation is observed. At low temperature the second term describes the dislocation motion.

Note that in silicon and gallium arsenide, the mean feature of the excitation-enhanced dislocation glide does not depend on the nature of the excitation source, either laser light or electron beam. In $\mathrm{Si}$, the study of the enhancement of dislocation motion either under photonic [18] or electronic [22] excitations led to similar values for activation energies both under excitation and without excitation. However photoplastic effects seems to affect screw dislocations and only one kind of $60^{\circ}$ dislocations (the $30 / 90$ one), whereas the results of the cathodoplasticity study do not exhibit such an asymmetry in the behaviour of $60^{\circ}$ dislocations. In the same way the behaviours of $\alpha$ dislocations in GaAs under either photonic [19] or electronic [3, 4] excitation are found to be very similar.

Photoplastic effects in GaAs have also been studied by microhardness tests followed by laser illumination $[23,24]$. Although it is not possible with this technique to perform quantitative measurements of dislocation velocity, a different behaviour of $\alpha$ and $\beta$ dislocations has been reported. 
Referring to the discussion of paragraph 2, the characterization of the microscopic processes affected by excitation requires the determination of which condition of kink collision $(\mathrm{X}<<\mathrm{L})$ and no kink collision $(\mathrm{X}>>\mathrm{L})$ is actually realized in the above experiments. This is of course not possible with the corresponding techniques. Maeda and Takeuchi [7] presume that the condition $X>>L$ is realized under experimental conditions, due to the fact that moving dislocations are probably bent and consequently the length of the rectilinear segments is rather short, but this is only speculative. In situ deformation experiments in a transmission electron microscope, as described below, give further information on the behaviour of dislocations under excitation at a microscopic scale.

3.2. Electron beam irradiation enhanced dislocation glide in semiconductors, as studied by transmission electron microscopy

The experimental procedure is as follows : macroscopic samples are first prestrained in order to introduce in the crystal a sufficiently high density of fresh dislocations. Tensile microsamples cut from the predeformed samples and thinned to electron transparency, are glued on a special tensile holder and stretched at elevated temperature. Dislocation motion is recorded in a videotape through a camera and an image intensifier.

Few experiments have been performed sofar to characterize the cathodoplastic effect. In GaAs, Maeda et al [25] have studied the glide behaviour of a dislocation loop expanding under an applied stress in the temperature range $370-800 \mathrm{~K}$. At constant beam intensity, the enhancement factor $\mathrm{v}_{\mathrm{i}} / \mathrm{v}_{\mathrm{d}}$ for $\alpha$ dislocations was observed to increase with lowering temperature (below $\sim 550 \mathrm{~K}$ ). The reduction in activation energy determined from this experiment was equal to the experimental value of $\Delta \mathrm{E}$ obtained for $\alpha$ dislocations in the scanning electron microscope measurements. No information is given in the paper on the velocity regime (length effect or not). Note that a recent study of dislocation mobility in GaAs [15] shows that the velocity of screw dislocations is proportional to their length up to $\mathrm{L} \simeq 3 \mu \mathrm{m}$ and that the enhancement factor at $623 \mathrm{~K}$ is very weak, in good agreement with these results.

The present authors have studied the cathodoplastic effects in single crystals of sphalerite $\mathrm{ZnS}$, in the temperature range $300-450 \mathrm{~K}[17,26,27]$.

During in situ straining experiments (foil surface (011); tensile axis [211], we have observed the glide of many individual dislocations. The movement of dislocations was observed to be viscous in all the studied temperature and intensity ranges. This feature strongly suggests that the dislocation motion is controlled by the Peierls mechanism, even in the enhancement regime. Many dislocations sources were observed. Fig. 2 shows a single-ended dislocation source which rotates counterclockwise, the screw direction being almost parallel to the slip traces. The local shear stress, evaluated from the radius of curvature of the dislocation line, is found to be equal to $40 \pm 12 \mathrm{MPa}$.

In this compound, measurements of dislocation velocity were made for each straight moving segment, as a function of its length $\mathrm{L}$ and electron beam intensity I. In all the studied temperature range and electron beam intensity range $(35-5600 \mathrm{~A} / \mathrm{m} 2)$ the dislocation velocity v varies linearly with $L$. This is illustrated in fig. 3 for the source displayed in fig. 2. Note that the $v(L)$ line do not intercept the $\mathrm{L}$ axis at the origin, but at some length $\mathrm{L}_{0} ; \mathrm{L}_{0}$ is probably related to the gradual transition between rectilinear parts of different character, which results in an accumulation of kinks at both ends of the moving segment, so that the effective length of the rectilinear segment is $\left(\mathrm{L}-\mathrm{L}_{0}\right)$. No saturation in dislocation velocity was observed in the studied length range, which is consistent with a high mobility of kinks along the dislocation line. 

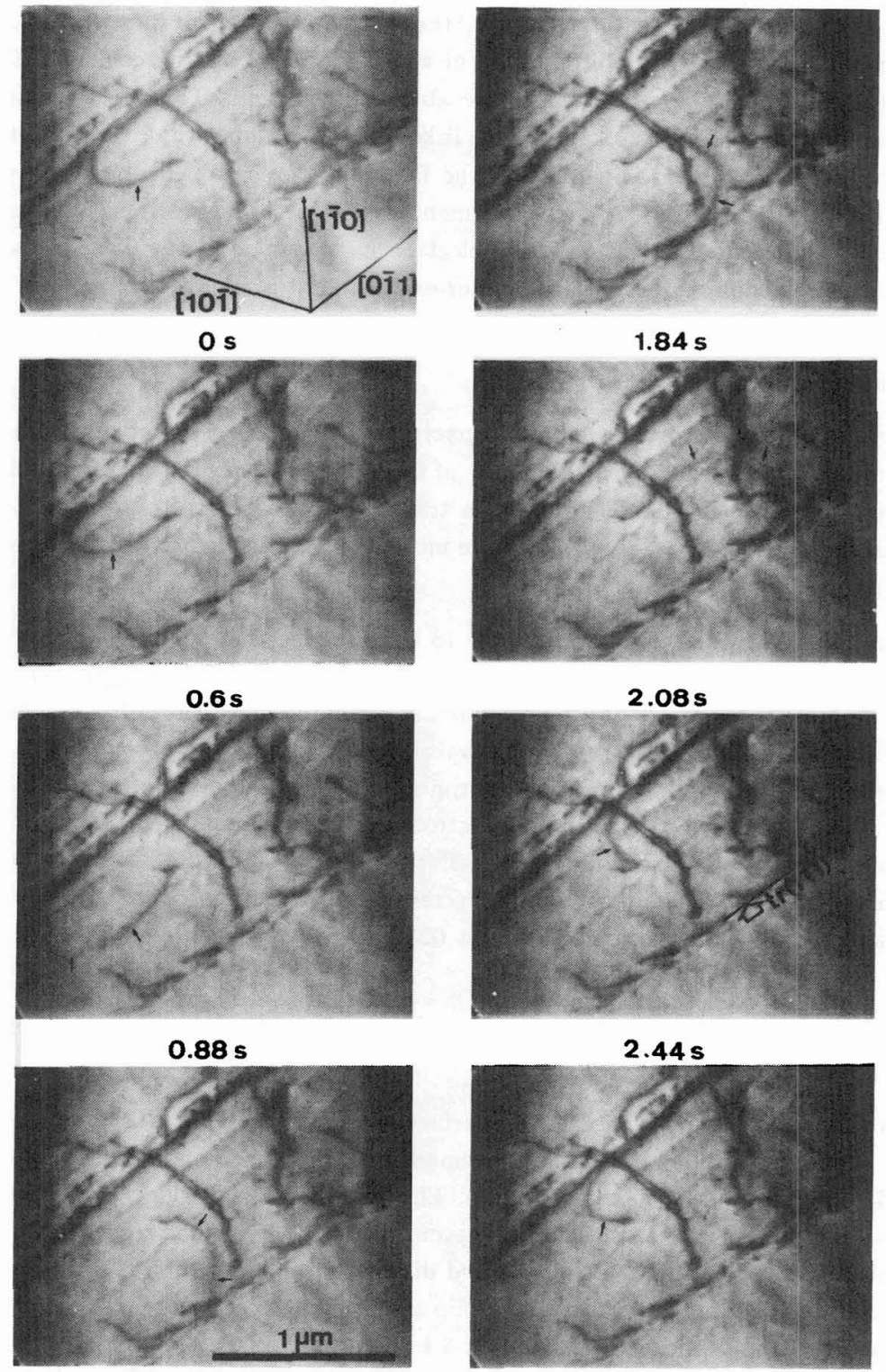

$1.32 \mathrm{~s}$

$2.72 \mathrm{~s}$

Fig. 2 : Dislocation source in $\mathrm{ZnS}$; the successive positions of the dislocation are arrowed. $\mathrm{T}=390 \mathrm{~K}$; $\mathrm{I}=5600 \mathrm{~A} / \mathrm{m}^{2} ; \tau=40 \pm 12 \mathrm{MPa}$. 


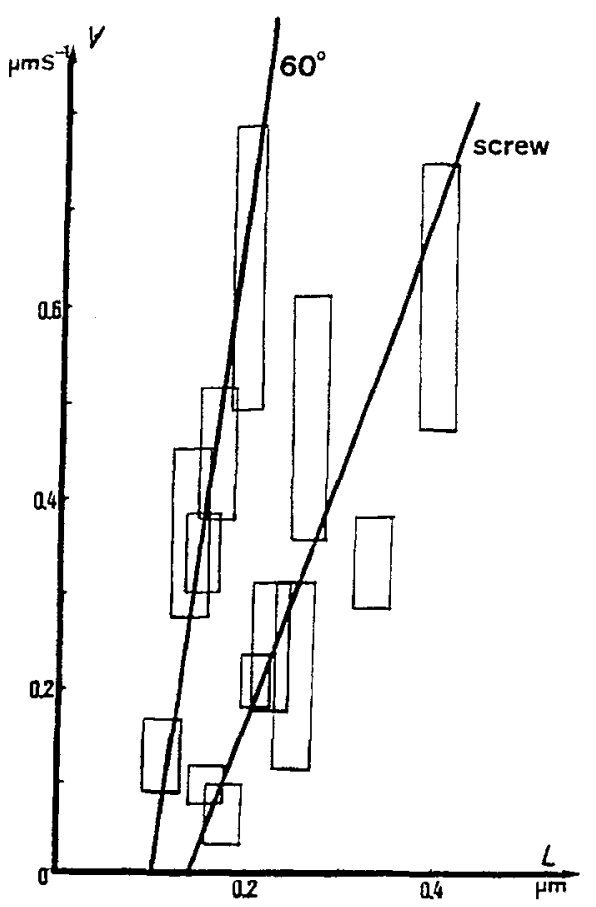

Fig. 3: Velocity of dislocations versus their length. $\mathrm{ZnS} . \mathrm{T}=390 \mathrm{~K} ; \mathrm{I}=5600 \mathrm{~A} / \mathrm{m}^{2}$; $\tau=40 \pm 12 \mathrm{MPa}$.

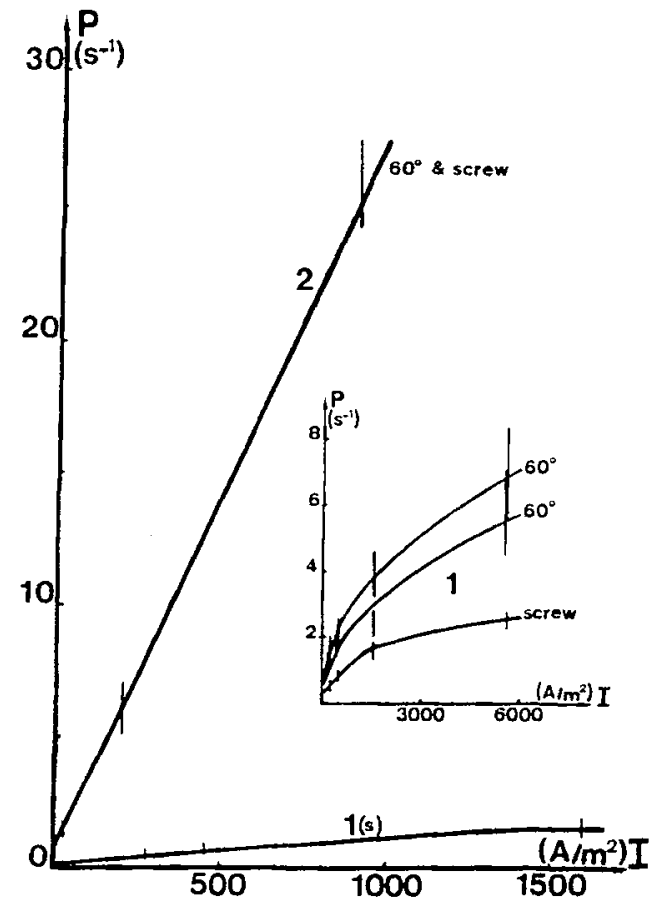

Fig. 4 : Slope of the v(L) curve versus electron beam intensity. ZnS. $\tau=40 \pm 12 \mathrm{MPa}$. (1) $\mathrm{T}=390 \mathrm{~K}$; (2) $\mathrm{T}=450 \mathrm{~K}$.

Moreover the dislocation velocity is strongly enhanced by electron excitation, as can be seen from fig. 4 which reports the variation of the slope $P$ of the $v(L)$ curve versus $I$ at $390 \mathrm{~K}$ and $450 \mathrm{~K}$. For $\mathrm{I} \leq 800 \mathrm{~m} 2, \mathrm{P}$ varies linearly with $\mathrm{I}$ at both temperatures, so that the dislocation velocity under excitation may be expressed as $v_{i}=v_{i o}^{\prime \prime}(T, r) L I$. This agrees qualitatively with the results reported in the previous section. For higher beam intensities $\mathrm{P}(\mathrm{I})$ at $390 \mathrm{~K}$ deviates from a linear relationship and a better fit of the experimental results is obtained by considering a logarithmic dependence of $P$ with I.

These results provide for the first time evidence of an enhancement of dislocation glide by an electron excitation, in the "length-effect" regime of velocity ( $\mathrm{X}>>L$, no kink collision). They indicate that the friction forces are higher without excitation, since dislocations are considerably slowed down as the electron beam intensity is reduced. 
Contrary to what is observed in III-V compounds the asymmetry in dislocation velocity is rather weak, both kind of $60^{\circ}$ dislocations being slightly more mobile than the screws at $390 \mathrm{~K}$. (Note that in the absence of suitable chemical reagent, the polarity of the crystal is not known; consequently the $\alpha(\beta)$ character of moving dislocations cannot be determined).

In some cases, dislocations move in their dissociated form. Preliminary measurements indicate that the behaviour of partial dislocations under electron excitation is similar to that of perfect ones.

\section{THE MECHANISM OF EXCITATION-ENHANCED DISLOCATION MOTION [7]}

A trivial cause of the enhancement may be the heating of the crystal due to the irradiation. Several investigators have checked this point $[3,4,18,24]$, either experimentally or theoretically, and have shown that the heating effect can be ruled out as the cause of enhancement.

Quite different models have been proposed to account for the features of the excitation-enhanced dislocation motion. It has been considered that the excitation effect has the same origin as the doping effect, i.e. the dislocation velocity is modified as the result of a change of the effective Fermi level in the crystal [28]. However Maeda and Takeuchi [3,4] were the first to point out that the excitation-enhanced dislocation motion shows features similar to those expected from the recombination-enhanced defect motion mechanism [29,30]. In this mechanism, the excitation induces an excess amount of minority carriers that are captured on the electronic energy level associated with the defect; subsequent capture of a majority carrier completes the recombination process. The energy released upon carrier capture is transferred non radiatively to the defect, in the form of vibrational energy. Finally, the induced lattice vibrations greatly enhance defect reactions such as movement of the defect itself or production of a new one, through reduction of activation energy.

Theoretical analysis $[29,30]$ shows that the rate of defect motion under excitation is given by :

$$
\mathrm{R}=\mathrm{r}_{\mathrm{o}} \exp -\frac{\mathrm{U}}{\mathrm{kT}}+\eta \mathrm{r}_{\mathrm{e}} \exp -\frac{\mathrm{U}-\Delta \mathrm{U}}{\mathrm{kT}}
$$

The first term corresponds to the ordinary thermal processes (prefactor $r_{o}$, activation energy U). The second term is the extra defect motion caused by the capture process : $\Delta U$ is closely related to the energy released upon carrier capture [30], $\eta<1$ is an efficiency factor and $r_{e}$ the recombinaison rate, which is proportional to the excitation intensity at low injection levels, but tends to saturate at high injection levels [30]; $\Delta U$ is independant of $r_{e}$.

All the observed features of the excitation-enhanced dislocation glide are consistent with the above equation (paragraph 3). This justifies the description of the effect in the general frame of the recombination-enhanced defect motion mechanism. We have now to consider how the microscopical mechanisms of dislocation motion in semiconductors, as described in paragraph 2 , are affected by non radiative recombination [7].

Maeda and Takeuchi [7] suggest that electronic states associated with straight portions of dislocations and kinks are different from each other, because these sites have different atomic structures. They assume that non radiative recombination of injected carriers could occur at both of these electronic levels; the energies released upon capture being $\Delta E_{s}$ for straight segment sites and $\Delta E_{k}$ for kink sites respectively. This means that :

$-\Delta E_{s}$ assists the initial kink pair formation

- $\Delta \mathrm{E}_{\mathbf{k}}$ assists the kink migration process.

Maeda and Takeuchi have analysed what changes are then brought about in dislocation motion. Three cases have to be considered : 
(1) Recombination-enhancement occurs at both straight dislocation and kink sites (i.e. it assists both kink pair formation and kink migration).

(2) Recombination-enhancement occurs only at straight dislocation sites (i.e. it assists only kink pair formation).

(3) Recombination-enhancement occurs only at kink sites (i.e. it assists only kink migration).

Their results are summarized in Table 1.

Table 1 : Intensity dependence of the velocity (at low injection level) and reduction in activation energy for excitation-enhanced dislocation motion [7]. (SD : straight dislocation site, $\mathrm{K}$ : kink site).

\begin{tabular}{|c|c|c|c|}
\hline $\begin{array}{c}\text { Velocity } \\
\text { Regime }\end{array}$ & $\begin{array}{c}\text { Site of } \\
\text { recombination }\end{array}$ & $\begin{array}{c}\text { Preexponential } \\
\text { factor }\end{array}$ & $\begin{array}{c}\text { Reduction in } \\
\text { activation energy }\end{array}$ \\
\hline \multirow{3}{*}{$X<L($ Kink collision $)$} & $S D+K$ & $I$ & $1 / 2\left(\Delta E_{s}+\Delta E_{k}\right)$ \\
& $S D$ & $I^{1 / 2}$ & $1 / 2 \Delta E_{s}$ \\
& $K$ & $I^{1 / 2}$ & $1 / 2 \Delta E_{k}$ \\
\hline \multirow{3}{*}{$X>L($ no kink collision $)$} & $S D+K$ & $I$ & $\Delta E_{s}$ \\
& $S D$ & $I$ & $\Delta E_{s}$ \\
& $K$ & no effect & no effect \\
\hline
\end{tabular}

It is seen that three cases exhibit the experimental feature of linear dependence on radiation intensity. To characterize the reduction in activation energy for dislocation glide, we have to know which velocity regime (kink collision or not) controls the dislocation motion in the experimental conditions. In situ deformation experiments, as described above, have shown that, at least in $\mathrm{ZnS}$, the enhancement occurs in the "length effect" regime of velocity (i.e. $X>>L$ ). That means that, according to the model, the assistance of kink migration, even if it occurs, does not contribute to the reduction in activation energy for dislocation motion. The observed reduction in activation energy is given by $\Delta E_{\mathbf{s}}$ and is therefore interpreted as an energy difference between an electronic energy level associated with a straight portion of dislocation and a relevant band edge. For the observations performed at a semi-microscopical scale, the conclusions are not so clear-cut, since the velocity regime is not unambiguously determined. As pointed out above, Maeda and Takeuchi [7] suggested that in these experiments the condition $\mathrm{X}>>\mathrm{L}$ was actually realized, but no definite proof was given. However, it should be noted that, in GaAs and InP, the activation energy for dislocation motion without excitation $\left(\mathrm{E}_{d}\right)$ as determined from scanning electron microscope experiments, is very close to the activation energy in the "length effect" regime $\left(F_{k p}+W_{m}\right)$, as determined by in situ straining experiments, in the temperature range when the enhancement does not occur $[15,31]$. This is consistent with the statement that the condition $X>>L$ was actually realized in the experiments performed at a semi-microscopical scale. Table 2 gives the activation energies evaluated from the various experimental approaches. As indicated above, the reduction in activation energy corresponds to the electronic energy level associated with the dislocation sites. A further confirmation is given by the results of recent intermittent loading experiments, which provide evidence that only the kink formation process in enhanced by electron excitation in GaAs [32]. 
Table 2 : Activation energies (in $\mathrm{eV}$ ) evaluated from the experiments :

$-\left(F_{k p}+W_{m}\right)_{d, i}$ :activation energies in the length effect regime, as determined by in situ straining experiments, without and under excitation, respectively.

$-\mathrm{E}_{\mathrm{d}, \mathrm{i}}$ : Activation energies as determined from scanning electron microscopy experiments.

Typical error bars are $\pm 0.1 \mathrm{eV}$.

\begin{tabular}{|c|c|c|c|c|c|}
\hline $\begin{array}{c}\text { Materials } \\
\text { (dislocation type) }\end{array}$ & $\left(F_{k p}+W_{m}\right)_{d}$ & $\left(F_{k p}+W_{m}\right)_{i}$ & $E_{d}$ & $E_{i}$ & $\Delta E$ \\
\hline$G a A s(\alpha)$ & & & 1.0 & $0.3 \quad[4]$ & 0.7 \\
\hline$G a A s(\beta)$ & $1.6 \quad[15]$ & & 1.7 & $0.6[4]$ & 1.1 \\
\hline GaAs(screw) & $1.6 \quad[15]$ & & 1.7 & $0.6[4]$ & 1.1 \\
\hline$G a P$ & & & 1.5 & 0.4 [21] & 1.1 \\
\hline $\operatorname{InP} P(\beta)$ & $1.5 \quad[31]$ & & 1.6 & $0.7 \quad[5]$ & 0.9 \\
\hline$Z n S(60)$ & 1.0 & 0.5 & & & 0.5 \\
\hline$Z_{n} S($ screw $)$ & 1.0 & 0.7 & & & 0.3 \\
\hline
\end{tabular}

\section{CONCLUSION}

In a variety of semiconductors, injection of minority carriers causes an enhancement of dislocation motion. Experimental features are consistent with the current models of dislocation motion in semiconductors and can be interpreted in the frame of the recombination enhanced defect motion theory. Particularly, the experiments performed at a microscopic scale (in situ deformation in a transmission electron microscope) provides conclusive evidence that dislocation motion under electron excitation is still controlled by the Peierls mechanism and that enhancement of dislocation velocity occurs in the so-called "length-effect" regime (no kink collision). A theoretical analysis led to the conclusion that the observed reduction in activation energy should correspond to the electronic energy levels associated with dislocations (and not with kinks) in the band gap. However it is not possible to specify whether it is the energy from the valence band edge or from the conduction band edge.

\section{REFERENCES}

1. Osip'Yan Yu.A., Petrenko V.F., Zaretskii A.V. and Withworth R.W., Adv. Phys., 35 (1986) 115

2. Couderc J.J., Levade C. and Kara A., Revue Phys. Appl., 25 (1990) 1129

3. George A. and Rabier J., Revue Phys. Appl., 22, (1987) 941

4. Maeda K., Sato M., Kubo A. and Takeuchi S., J. Appl. Phys., 54 (1983) 161

5. Maeda K. and Takeuchi S., J. Physique 44 (1983) C4-375

6. For Example : Petroff P.N., J. Physique, 40 (1979) C6-201

7. Maeda N. and Takeuchi S., Inst. Phys. Conf. Ser., 104 (1989) 303

8. Hirth J.P. and Lothe J., Theory of Dislocations, McGraw-Hill, New-York (1968) p. 493

9. Alexander H., Haasen P., Labusch R.and Schroter W., J. Physique, 40 (1979) C6-foreword

10. Wessel K. and Alexander H., Phil. Mag., 35 (1977) 1523

11. Alexander H. in Dislocations 84, P. Veyssière, L. Kubin and J. Castaing eds., CNRS Paris (1984), p. 283 
12. Steinhardt H. and Schafer S., Acta Met., 19 (1971) 65

13. Ninomiya T., J. Physique, 40 (1979) C6-143

14. Fnaiech M., Reynaud F., Couret A. and Caillard D., Phil. Mag., A55 (1987) 405

15. Caillard D., Clement N., Couret A., Androussi Y., Lefebvre A. and Vanderschaeve G。, inst. Phys. Conf. Ser., 87 (1987) $361 ; 100$ (1989) 403

16. Lefevbre A., Androussi Y. and Vanderschaeve G., Phil. Mag. Lett., 56 (1987) 135

17. Levade C., Vanderschaeve G., Couderc J.J., Faress A. and Caillard D., Microscopy of Semiconducting Materials VII, Oxford (1991), in the press

18. Kuesters K.H. and Alexander H., Physica $116 B$ (1983) 594

19. Mdivanyan B.E. and Shikhsaidov M.Sh., Phys. Stat. Sol. (a) 107 (1988) 131

20. Takeuchi S. and Maeda K., in Dislocations 84, P. Veyssière, L. Kubin and J. Castaing eds, CNRS Paris, (1984) p. 331

21. Maeda N. and Takeuchi S., Japanese J. Appl. Phys., 29 (1990) 1151

22. Maeda N., Kimura K. and Takeuchi S., Bull. Acad. Sci. USSR, Physical Series 51 (1987) 93

23. Fujita S., Maeda K. and Hyodo S., Phys. Stat. Sol. (a) 109 (1988), 383

24. Depraetère E., Vignaud D., Farvacque J.L., Sieber B. and Lefebvre A., Phil. Mag., A61 (1990) 893

25. Maeda K., Suzuki K., Ichihara M. and Takeuchi S., J. Appl. Phys., 56 (1984) 554

26. Levade C., Couderc J.J., Caillard D. and Couret A., in Electron Microscopy in Plasticity and Fracture Research of Materials, U. Messerschmidt et al. eds, Akademie Verlag, Berlin (1990) p. 199 27. Levade C., Couderc J.J., Vanderschaeve G., Caillard D. and Couret A., E-MRS Fall Meeting : Analytical techniques for the characterization of compound semiconductors, Strasbourg (1990); Appl. Surf. Science, in the press

28. Belyavskii V.I., Darinskii B.M. and Sviridov V.V., Soviet Phys. Solid State, 27 (1985) 658

29. Weeks J.D., Tully J.C. and Kimmerling L.C., Phys. Rev., B 12 (1975) 3286

30. Sumi H., Phys. Rev., B, 29 (1984) 4616

31. Zafrany M., Voillot F., Peyrade J.P., Caillard D., Couret A. and Coquillé R., Phil. Mag. A (1991), in the press

32. Maeda K., Yamashita Y., Maeda N. and Takeuchi S., Mat Res. Soc. Symp., 184 (1990) 69. 